case studies where we are using our workflow to generate insights into big challenges in the hospital, i) a proof-of-concept surgery scheduling algorithm for improving theatre utilisation and reducing on-the-day cancellations and ii) an outpatient demand prediction model to understand the impact of international and private patients on diagnostic services in the hospital.

\section{FEMUR FRACTURES IN CHILDREN WITH CANCERS. WHAT COULD BE THE AETIOLOGY?}

${ }^{1}$ Jagath Ranasinghe, ${ }^{2}$ Mette Jorgensen. ${ }^{1}$ Clinical Fellow; ${ }^{2}$ Consultant Paediatric Oncologist

10.1136/archdischild-2020-gosh.111

Introduction Femur fractures in children are uncommon. Incidence of fractures in leukaemia is about $13.5 \%$ and is 6 fold higher than estimate. No prevalence data available on fractures among hospitalized children with cancer. Among the cancer patients femur fractures could be due to various reasons such as non-accidental injuries (NAI), osteoporosis and bone metastasis. We report two patients who had mid shaft femur fractures while receiving chemotherapy as in patients.

Case history Case 01, 17 months old girl diagnosed with Ewings sarcoma (EWSR1-FLI1 type-2) and paraplegia. Whilst receiving chemotherapy she was experienced acute swelling of her left thigh and diagnosed with a spiral femur fracture which was unrelated to disease. Case 02,4 year old boy with adrenal cortical tumour. During his treatment he sustained a spiral fracture of his left femur. Both these children sustained their fracture while inpatient and extensive review excluded a possible safeguarding issues.

Discussion Case 01 was non ambulatory and case 02 was ambulatory but less able. In multidisciplinary child protection meeting mechanism of the fracture of case 01 was not clear. Child had a habit of trying to bite her toe. But, mum noted she might have caused this when trying to keep her on her side and child refused/rotated - as she did not feel pain therefore the force is difficult to assess - No ill intention was meant. Case 02, he tried to get down from the bed when he falls which leads to the fracture. The conclusion is that there was no evidence of child abuse. Lack of supervision of caregivers was raised as an issue in both cases.

Conclusion Spiral femur fracture in a non-ambulatory child must always raise a concern of NAI. However prevalence of fractures of children with cancers in hospital need more evaluation as those are preventable.

\section{\begin{tabular}{|l|l}
\hline 112 & IMPROVING CLINICAL HANDOVER
\end{tabular}}

${ }^{1}$ Sevasti Konstantinidou, ${ }^{1}$ Nikita Patel, ${ }^{1}$ Jayaram Sivaraj, ${ }^{1}$ Manal Dhaiban, ${ }^{1}$ Stavros Loukogeorgakis, ${ }^{2}$ Ceri Jones. ${ }^{1}$ Great Ormond Street Hospital; ${ }^{2}$ GOSH

\subsection{6/archdischild-2020-gosh.112}

Background Handover is an important clinical skill combining knowledge, prioritisation and communication. This has been highlighted within the emergency working patterns essential for patient care during the pandemic. Effective handover can reduce the incidence of adverse events and benefit patients, clinicians and the hospital. The aim of this project is to assess and improve the quality of departmental verbal and written handover.
Methods A quality improvement method was used to assess the quality of verbal and written handover using verified opinion-based questionnaires for participants. The verbal handover was also assessed by measuring objective parameters including duration, participants and presenter type, number of patients discussed, situational awareness and task management, time pressure and distractions and teamwork factors.

Following the initial audit, standardised handover formats and a traffic light system were implemented to assist with prioritising patients. Results were compared before and after intervention with descriptive statistics.

Results 20 members of our department completed our pre intervention questionnaire and results showed that most participants were dissatisfied with the verbal handover and 50\% thought that this might result in adverse events. The disadvantages identified were lengthy duration, multiple interruptions and inappropriate team member leading handover. The objective assessment tool showed that areas of weakness were communication, accountability, task management and distractions. Following intervention with standardisation, objective measures remained stable but subjective responses improved. The satisfaction on general process, duration and person leading handover increased.

Conclusions 'Handover' is highlighted as an important clinical skill especially when working in unfamiliar team and shifts patterns. Implementing a standardised departmental handover has had a positive effect on the team and the process. We intend to continue to improve the handover process by implementing a standardised structure for written handover and continued integrated teaching highlighting communication, prioritisation and verbal handover.

\section{RESTRUCTURING OF PAEDIATRIC UROLOGY OUT- PATIENT WORKFLOW DURING THE COVID-19 PANDEMIC}

${ }^{1}$ Neetu Kumar, ${ }^{2}$ Francie Kaushal, ${ }^{1}$ Alexander Cho, ${ }^{1}$ Imran Mushtaq, ${ }^{1}$ Navroop Johal. ${ }^{1}$ Department of Paediatric Urology, Great Ormond Street Hospital; ${ }^{2}$ Great Ormond Street Hospital

\subsection{6/archdischild-2020-gosh.113}

Aim To audit restructuring of out-patient clinics at GOSH during the COVID-19 pandemic with the aim of evaluating service delivery and business continuity, focussing specifically on the utility of telephonic clinics.

Methods We focussed on paediatric urology out-patient clinics at GOSH. The electronic patient records were accessed to acquire numbers of clinics and patients booked, total face-toface and telephonic clinic attendances and DNA (did not attend) percentages. Three- monthly blocks were evaluated: April-June 2020, spanning the first peak of the pandemic, April-June 2019 in the preceding year to match activity, and then subsequently the period July-September 2020 to evaluate how the services settled in the post-pandemic phase. The audit was registered with the GOSH Clinical Audit Department. Significance of difference between study groups was examined by Student's t-test.

Results Total of 95 clinics were booked with 874 patients during the pandemic block with $80.6 \%(\mathrm{~N}=705)$ done telephonically. The DNA rate during this time was $11.5 \%$. Comparingly, in the same calendar period of 2019, 79 clinics had been booked with all $(100 \%, \mathrm{~N}=848)$ patients seen face- 